\title{
The Association Between Discharge Before Noon and Length of Stay in Medical and Surgical Patients
}

\author{
Alvin Rajkomar, MD*, Victoria Valencia, MPH, Maria Novelero, MA, MPA, Michelle Mourad, MD, Andrew Auerbach, MD, MPH
}

Department of Medicine, Division of Hospital Medicine, University of California, San Francisco, San Francisco, California.

BACKGROUND: Discharging patients before noon is a key approach to improving bed utilization. Few data exist to describe whether patients are discharged earlier or their stay is extended to allow for an early discharge the next day.

OBJECTIVE: To determine if a discharge before noon (DCBN) is associated with length of stay (LOS).

DESIGN/SETTINGS/PATIENTS: Retrospective analysis of data from adult medical and surgical discharges from a single academic center from July 2012 through April 2015. We used a multivariable generalized linear model to evaluate the association between DCBN and LOS.

RESULTS: Of 38,365 hospitalizations, 6484 (16.9\%) were discharged before noon, and the median LOS was 3.7 days.
After adjustment, DCBN was associated with a longer LOS (adjusted odds ratio [OR]: 1.043, 95\% confidence interval [CI]: 1.003-1.086). The association between longer LOS and DCBN was more pronounced in patients admitted emergently ( $\mathrm{n}=14,192,37 \%)$ (adjusted OR: $1.14,95 \% \mathrm{Cl}$ : 1.033-1.249).

CONCLUSIONS: Although we cannot discern whether discharges were delayed to achieve discharge before noon, earlier discharge was associated with a longer LOS, particularly among emergent admissions. Journal of Hospital Medicine 2015;11:859-861. (C) 2015 Society of Hospital Medicine
Slow hospital "throughput"- the process whereby a patient is admitted, placed in a room, and eventually discharged-can worsen outcomes if admitted patients are boarded in emergency rooms or postanesthesia units. ${ }^{1}$ One potential method to improve throughput is to discharge patients earlier in the day, ${ }^{2}$ freeing up available beds and conceivably reducing hospital length of stay (LOS).

To quantify throughput, hospitals are beginning to measure the proportion of patients discharged before noon (DCBN). One study, looking at discharges on a single medical floor in an urban academic medical center, suggested that increasing the percentage of patients discharged by noon decreased observed-toexpected LOS in hospitalized medicine patients, ${ }^{3}$ and a follow-up study demonstrated that it was associated with admissions from the emergency department occurring earlier in the day. ${ }^{4}$ However, these studies did not adjust for changes in case mix index (CMI) and other patient-level characteristics that may also have affected these outcomes. Concerns persist that more efforts to discharge patients by noon could inad-

*Address for correspondence and reprint requests: Alvin Rajkomar, MD, 533 Parnassus Ave., Suite 125, San Francisco, CA 94143-0131; Telephone: 415-476-1528; Fax: 415-502-1976; E-mail: alvin.rajkomar@ucsf. edu

Additional Supporting Information may be found in the online version of this article.

Received: July 30, 2015; Revised: November 24, 2015; Accepted: November 29, 2015

2015 Society of Hospital Medicine DOI 10.1002/jhm.2529

Published online in Wiley Online Library (Wileyonlinelibrary.com). vertently increase LOS if staff chose to keep patients overnight for an early discharge the following day.

We undertook a retrospective analysis of data from patients discharged from a large academic medical center where an institution-wide emphasis was placed on discharging more patients by noon. Using these data, we examined the association between discharges before noon and LOS in medical and surgical inpatients.

\section{METHODS}

\section{Site and Subjects}

Our study was based at the University of California, San Francisco (UCSF) Medical Center, a 400-bed academic hospital located in San Francisco, California. We examined adult medical and surgical discharges from July 2012 through April 2015. Patients who stayed less than 24 hours or more than 20 days were excluded. Discharges from the hospital medicine service and the following surgical services were included in the analysis: cardiac surgery, colorectal surgery, cardiothoracic surgery, general surgery, gynecologic oncology, gynecology, neurosurgery, orthopedics, otolaryngology, head and neck surgery, plastic surgery, thoracic surgery, urology, and vascular surgery. No exclusions were made based on patient status (eg, observation vs inpatient). UCSF's institutional review board approved our study.

During the time of our study, discharges before noon time became an institutional priority. To this end, rates of DCBN were tracked using retrospective data, and various units undertook efforts such as informal afternoon meetings to prompt planning for the next morning's discharges. These efforts did not 
differentially affect medical or surgical units or emergent or nonemergent admissions, and no financial incentives or other changes in workflow were in place to increase DCBN rates.

\section{Data Sources}

We used the cost accounting system at UCSF (Enterprise Performance System Inc. [EPSI], Chicago, IL) to collect demographic information about each patient, including age, sex, primary race, and primary ethnicity. This system was also used to collect characteristics of each hospitalization including LOS (calculated from admission date time and discharge date time), hospital service at discharge, the discharge attending, discharge disposition of the patient, and the CMI, a marker of the severity of illness of the patient during that hospitalization. EPSI was also used to collect data on the admission type of all patients, either emergent, urgent, or routine, and the insurance status of the patient during that hospitalization.

Data on time of discharge were entered by the discharging nurse or unit assistant to reflect the time the patient left the hospital. Using these data, we defined a before-noon discharge as one taking place between 8:00 AM and 12:00 PM.

\section{Statistical Analysis}

Wilcoxon rank sum test and $\chi^{2}$ statistics were used to compare baseline characteristics of hospitalizations of patients discharged before and after noon.

We used generalized linear models to assess the association of a discharge before noon on the LOS with gamma models. We accounted for clustering of discharge attendings using generalized estimating equations with exchangeable working correlation and robust standard errors. After the initial unadjusted analyses, covariates were included in the adjusted analysis if they were associated with an LOS at $P<$ 0.05 or reasons of face validity. These variables are shown in Table 1. Because an effort to increase the discharges before noon was started in the 2014 academic year, we added an interaction term between the date of discharge and whether a discharge occurred before noon. The interaction term was included by dividing the study period into time periods corresponding to sequential 6-month intervals. A new variable was defined by a categorical variable that indicated in which of these time periods a discharge occurred.

We conducted a sensitivity analysis using propensity scores. The propensity score was based on demographic and clinical variables (as listed in Table 1) that exhibited $P<0.2$ in bivariate analysis between the variable and being discharged before noon. We then used the propensity score as a covariate in a generalized linear model of the LOS with a gamma distribution and with generalized estimating equations as described above.

\begin{tabular}{|c|c|c|c|}
\hline & $\begin{array}{l}\text { Discharged } \\
\text { Before Noon }\end{array}$ & $\begin{array}{l}\text { Discharged } \\
\text { After Noon }\end{array}$ & $P$ Value \\
\hline Median LOS (IQR) & $3.4(2.2-5.9)$ & $3.7(2.3-6.3)$ & $<0.0005$ \\
\hline Median CMI (IQR) & $1.8(1.1-2.4)$ & $1.7(1.1-2.5)$ & 0.006 \\
\hline \multicolumn{4}{|l|}{ Service type, N (\%) } \\
\hline Hospital medicine & $1,919(29.6)$ & $11,290(35.4)$ & \\
\hline Surgical services & $4,565(70.4)$ & $20,591(64.6)$ & $<0.0005$ \\
\hline Discharged before noon, N (\%) & $6,484(16.9)$ & $31,881(83.1)$ & \\
\hline \multicolumn{4}{|l|}{ Discharged on weekend, N (\%) } \\
\hline Yes & $1,543(23.8)$ & $7,411(23.3)$ & \\
\hline № & $4,941(76.2)$ & $24,470(76.8)$ & 0.34 \\
\hline \multicolumn{4}{|l|}{ Discharge disposition, N (\%) } \\
\hline Home with home health & $748(11.5)$ & $5,774(18.1)$ & \\
\hline Home without home health & $3,997(61.6)$ & $17,862(56.0)$ & \\
\hline SNF & $837(12.9)$ & $3,082(9.7)$ & \\
\hline Other & $902(13.9)$ & $5,163(16.2)$ & $<0.0005$ \\
\hline \multicolumn{4}{|l|}{ 6-month interval, N (\%) } \\
\hline July-December 2012 & $993(15.3)$ & $5,596(17.6)$ & \\
\hline January-June 2013 & $980(15.1)$ & $5,721(17.9)$ & \\
\hline July-December 2013 & $1,088(16.8)$ & $5,690(17.9)$ & \\
\hline January-June 2014 & $1,288(19.9)$ & $5,441(17.1)$ & \\
\hline July-December 2014 & $1,275(19.7)$ & $5,656(17.7)$ & \\
\hline January-April 2015 & $860(13.3)$ & $3,777(11.9)$ & $<0.0005$ \\
\hline \multicolumn{4}{|l|}{ Age category, N (\%) } \\
\hline 18-64 years & $4,177(64.4)$ & $20,044(62.9)$ & \\
\hline $65+$ years & $2,307(35.6)$ & $11,837(37.1)$ & 0.02 \\
\hline Male, N (\%) & $3,274(50.5)$ & $15,596(48.9)$ & \\
\hline Female, N (\%) & $3,210(49.5)$ & $16,284(51.1)$ & 0.06 \\
\hline \multicolumn{4}{|l|}{ Race, N (\%) } \\
\hline White or Caucasian & $4,133(63.7)$ & $18,798(59.0)$ & \\
\hline African American & $518(8.0)$ & $3,020(9.5)$ & \\
\hline Asian & $703(10.8)$ & $4,052(12.7)$ & \\
\hline Other & $1,130(17.4)$ & $6,011(18.9)$ & $<0.0005$ \\
\hline \multicolumn{4}{|l|}{ Ethnicity, N (\%) } \\
\hline Hispanic or Latino & $691(10.7)$ & $3,713(11.7)$ & \\
\hline Not Hispanic or Latino & $5,597(86.3)$ & $27,209(85.4)$ & \\
\hline Unknown/declined & $196(3.0)$ & $959(3.0)$ & 0.07 \\
\hline \multicolumn{4}{|l|}{ Admission type, N (\%) } \\
\hline Elective & $3,494(53.9)$ & $13,881(43.5)$ & \\
\hline Emergency & $2,047(31.6)$ & 12,145 (38.1) & \\
\hline Urgent & $889(13.7)$ & $5,459(17.1)$ & \\
\hline Other & $54(0.8)$ & $396(1.2)$ & $<0.0005$ \\
\hline \multicolumn{4}{|l|}{ Payor class, N (\%) } \\
\hline Medicare & $2,648(40.8)$ & $13,808(43.3)$ & \\
\hline Medi-Cal & $1,060(16.4)$ & $5,913(18.6)$ & \\
\hline Commercial & $2,633(40.6)$ & $11,242(35.3)$ & \\
\hline Other & $143(2.2)$ & $918(2.9)$ & $<0.0005$ \\
\hline
\end{tabular}

NOTE: Abbreviations: CMI, case mix index; IQR, interquartile range; LOS, length of stay; SNF, skilled nursing facility.

Finally, we performed prespecified secondary subset analyses of patients admitted emergently and nonemergently.

Statistical modeling and analysis was completed using Stata version 13 (StataCorp, College Station, TX).

\section{RESULTS}

\section{Patient Demographics and Discharge Before Noon}

Our study population comprised 27,983 patients for a total of 38,365 hospitalizations with a median LOS of 3.7 days. We observed 6484 discharges before noon $(16.9 \%)$ and 31,881 discharges after noon $(83.1 \%)$. 
The characteristics of the hospitalizations are shown in Table 1.

Patients who were discharged before noon tended to be younger, white, and discharged with a disposition to home without home health. The median CMI was slightly higher in discharges before noon (1.81, $P=0.006$ ), and elective admissions were more likely than emergent to be discharged before noon $(53.9 \%$ vs $31.6 \%, P<0.0005)$.

\section{Multivariable Analysis}

A discharge before noon was associated with a $4.3 \%$ increase in LOS (adjusted odds ratio [OR]: 1.043, 95\% confidence interval [CI]: 1.003-1.086), adjusting for CMI, the service type, discharge on the weekend, discharge disposition, age, sex, ethnicity, race, urgency of admission, payor class, and a full interaction with the date of discharge (in 6-month intervals). In preplanned subset analyses, the association between longer LOS and DCBN was more pronounced in patients admitted emergently (adjusted OR: 1.14, 95\% CI: 1.033-1.249) and less pronounced for patients not admitted emergently (adjusted OR: 1.03, 95\% CI: 0.988-1.074), although the latter did not meet statistical significance. In patients admitted emergently, this corresponds to approximately a 12-hour increase in LOS. The interaction term of discharge date and DCBN was significant in the model. In further subset analyses, the association between longer LOS and DCBN was more pronounced in medicine patients (adjusted OR: 1.116, 95\% CI: 1.014-1.228) than in surgical patients (adjusted OR: 1.030, 95\% CI: 0.9891.074), although the relationship in surgical patients did not meet statistical significance.

We also undertook sensitivity analyses utilizing propensity scores as a covariate in our base multivariable models. Results from these analyses did not differ from the base models and are not presented here. Results also did not differ when comparing discharges before and after the initiation of an attending only service.

\section{DISCUSSION AND CONCLUSION}

In our retrospective study of patients discharged from an academic medical center, discharge before noon was associated with a longer LOS, with the effect more pronounced in patients admitted emergently in the hospital. Our results suggest that efforts to discharge patients earlier in the day may have varying degrees of success depending on patient characteristics. Conceivably, elective admissions recover according to predictable plans, allowing for discharges earlier in the day. In contrast, patients discharged from emergent hospitalizations may have ongoing evolution of their care plan, making plans for discharging before noon more challenging.

Our results differ from a previous study, ${ }^{3}$ which suggested that increasing the proportion of beforenoon discharges was associated with a fall in observed-to-expected LOS. However, observational studies of DCBN are challenging, because the association between early discharge and LOS is potentially bidirectional. One interpretation, for example, is that patients were kept longer in order to be discharged by noon the following day, which for the subgroups of patients admitted emergently corresponded to a roughly 12-hour increase in LOS. However, it is also plausible that patients who stayed longer also had more time to plan for an early discharge. In either scenario, the ability of managers to utilize LOS as a key metric of throughput efforts may be flawed, and suggests that alternatives (eg, number of patients waiting for beds off unit) may be a more reasonable measure of throughput. Our results have several limitations. As in any observational study, our results are vulnerable to biases from unmeasured covariates that confound the analysis. We caution that a causal relationship between a discharge before noon and LOS cannot be determined from the nature of the study. Our results are also limited in that we were unable to adjust for day-to-day hospital capacity and other variables that affect LOS including caregiver and transportation availability, bed capacity at receiving care facilities, and patient consent to discharge. Finally, as a singlesite study, our findings may not be applicable to nonacademic settings.

In conclusion, our observational study discerned an association between discharging patients before noon and longer LOS. We believe our findings suggest a rationale for alternate approaches to measuring an early discharge program's effectiveness, namely, that the evaluation of the success of an early discharge initiative should consider multiple evaluation metrics including the effect on emergency department wait times, intensive care unit or postanesthesia transitions, and on patient reported experiences of care transitions.

Disclosures: Andrew Auerbach, MD, is supported by a K24 grant from the National Heart, Lung, and Blood Institute: K24HL098372. The authors report no conflicts of interest.

\section{References}

1. Bernstein SL, Aronsky D, Duseja R, et al. The effect of emergency department crowding on clinically oriented outcomes. Acad Emerg Med. 2009;16(1):1-10.

2. Centers for Medicare \& Medicaid Services, The Joint Commission. Specifications manual for national hospital inpatient quality measures [version 4.2b]. Oakbrook Terrace, IL: The Joint Commission; 2013.

3. Wertheimer B, Jacobs REA, Bailey M, et al. Discharge before noon: an achievable hospital goal. J Hosp Med. 2014;9(4):210-214.

4. Wertheimer B, Jacobs REA, Iturrate E, et al. Discharge before noon: effect on throughput and sustainability. J Hosp Med. 2015;10(10): 664-669. 